\title{
In-Situ, Real Time Observation of Operating Condition of Multi Emitters in Pulse Mode*
}

\author{
Hidekazu Murata, ${ }^{\dagger}$ Kentaro Sakai, Daisuke Tsubaki, and Hiroshi Shimoyama \\ Faculty of Science \& Technology, Meijo University, Tempaku-ku, Nagoya 468-8502, Japan \\ Kazuto Sakemura, Nobuyasu Negishi, and Atsushi Watanabe \\ Corporate REDD Laboratories, Pioneer Corporation, Tsurugashima, Saitama 350-2288, Japan \\ (Received 6 January 2010; Accepted 27 March 2010; Published 29 May 2010)
}

\begin{abstract}
We have developed an electron optical instrument for evaluation of multi emitters. The instrument is a versatile emission microscope and is capable of operating as a secondary electron emission microscope (SEEM), a photo electron emission microscope (PEEM) and a field electron emission microscope (FEEM) imaging modes. The most important feature of the instrument is the capability of simultaneous observation of SEEM and FEEM images as well as PEEM and FEEM images in real time and in-situ mode. The operating condition of the multi emitters can be observed in DC mode as well as pulse mode. Thus, the instrument enables us to obtain quantitative knowledge as to the percentage of actually working emitters out of the whole emitters and to evaluate the stability of the emission current from each individual working emitter. [DOI: 10.1380/ejssnt.2010.266]
\end{abstract}

Keywords: Electron emission measurements; Secondary electron emission microscopy (SEEM); Photo electron emission microscopy (PEEM); Field electron emission microscopy (FEEM); Nano-electronics and related devices;

\section{INTRODUCTION}

Multi emitters such as field emitter arrays (FEAs), a metal insulator semiconductor (MIS) structure device called HEED (high-efficiency electron emission device) [1] and carbon nanotubes (CNTs) have been attracting much attention because of their great potentialities for wide range of future applications. One of the most promising applications of multi emitters is their use as multi field emission sources for field emission displays (FEDs) or flat panel displays (FPDs). Multi emitters have recently been examined as an electron source for a high power traveling microwave tube (TWT) [2] for satellite communication. Multi emitters are also regarded as strong candidates for multi field emission sources for a multi-column, multibeam direct write electron beam lithography system [3, 4], because a high throughput, high resolution electron beam lithography system is strongly required as a substitute for a light optical lithography system in the next generation.

In spite of their important potentialities, there exist very few instruments to carry out systematic and quantitative studies for evaluating their emission characteristics and performances. Little quantitative knowledge has been obtained so far on the points as to (1) the percentage of actually working emitters out of the whole emitters, (2) reasons why some of the existing emitters are not working, (3) stability of the emission current from each individual working emitter, (4) reasons for instability and possible countermeasures against it if the emission current is unstable, and so on. The situation has motivated us to develop a novel electron optical instrument for evaluation of emission characteristics of multi emitters [5]. This paper describes the outline of the instrument and some recent experimental results.

*This paper was presented at 7th International Symposium on Atomic Level Characterizations for New Materials and Devices, The Westin Maui Resort \& Spa, Hawaii, U.S.A., 6-11 December, 2009.

†Corresponding author: hkmurata@ccmfs.meijo-u.ac.jp

\section{OUTLINE OF THE INSTRUMENT}

Figure 1 shows a schematic diagram of the electron optical instrument for evaluation of multi emitters. For detail of the region enclosed by dashed lines, see Fig. 2. The specimen (= multi emitters) is kept floating at a negative high potential of about $-10 \mathrm{kV}$, while the imaging lens system, which consists of objective lenses (OL and OL2), intermediate lenses (IL1 and IL2) and a projector lens $(\mathrm{PL})$, is grounded on the earth.

The instrument is a versatile emission microscope, in which a magnified image of specimen surface can be obtained by means of electrons emitted from the specimen itself floating at a negative high potential. The instrument is equipped with a beam illumination system that irradiates the specimen with electron beam or UV light for obtaining secondary (or reflected) electrons or photo electrons emitted from specimen surface. Field emitted electrons can also be obtained from emission sites of the specimen by controlling the extraction voltage (or drive voltage) applied through the specimen stage. Those electrons emitted from the specimen surface are used to form the magnified image of the specimen surface onto the entrance plane of the multi channel plate (MCP) by means of the imaging lens system. Thus, the instrument is capable of operating as a secondary electron emission microscope (SEEM), a photo electron emission microscope (PEEM) and a field electron emission microscope (FEEM). In SEEM imaging mode, the incident energy of electron beam is generally a several tens of $\mathrm{eV}$. Therefore, secondary electrons are predominantly emitted from the specimen surface. The operation mode of SEEM is almost same as that of low energy electron microscope (LEEM) mode, where the incident energy of electron beam is generally used at below about $10 \mathrm{eV}$, and the magnified image of the specimen surface is formed by means of diffracted electrons elastically-scattered on the specimen surface. The most important feature of the instrument is the capability of simultaneous observation of SEEM and FEEM images as well as PEEM and FEEM images in real time and in-situ mode, as described later. 


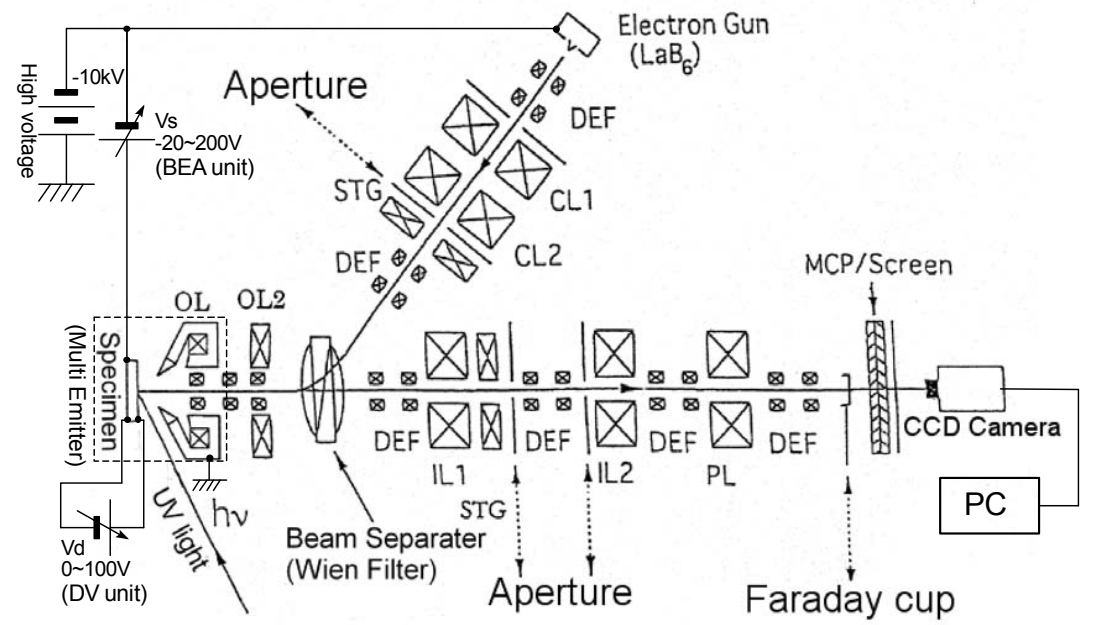

FIG. 1: Schematic diagram of electron optical instrument for evaluation of multi emitters. For detail of the region enclosed by dashed lines, see Fig. 2.
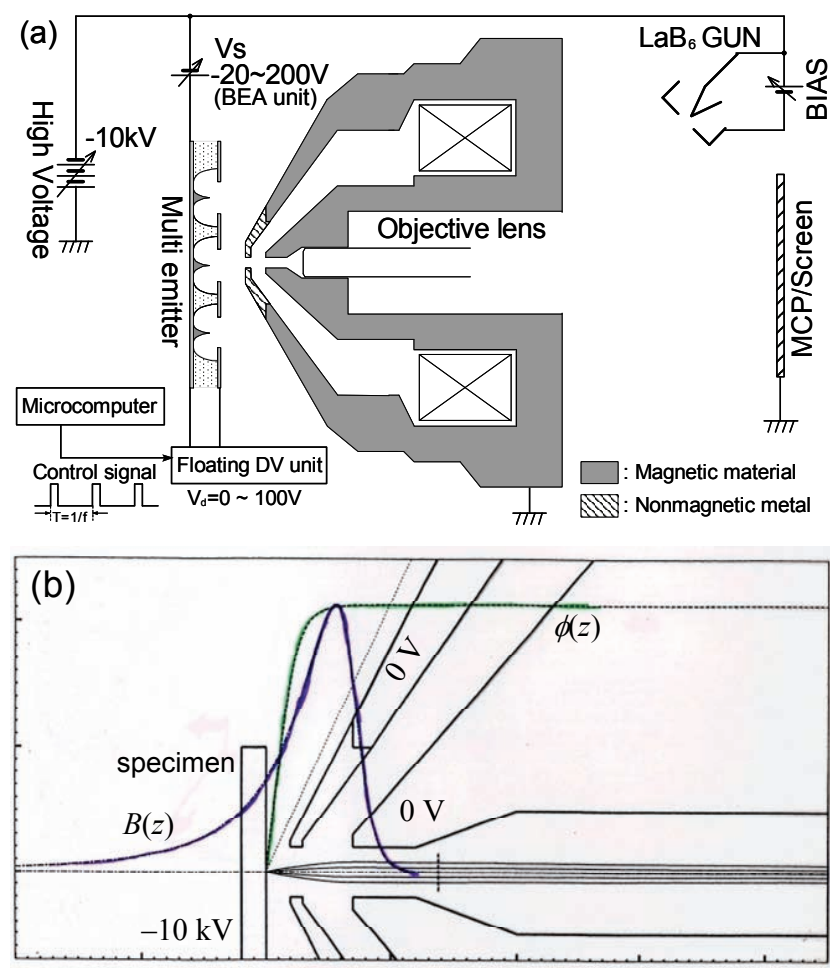

FIG. 2: (a) Schematic diagram of the vicinity of a specimen and an objective lens, and computer controlled floating power supply. The floating DV unit is the power supply for driving multi emitters and is controlled by a microcomputer. The multi emitters can be driven in DC mode as well as in pulse mode. (b) Axial potential $\phi(z)$ and magnetic field $B(z)$ in the vicinity of a specimen and an objective lens.

Special attention has been paid to the design of the objective lens OL, which determines the ultimate performance (i.e., the spatial resolution) of the instrument. The objective lens field of the emission microscope normally consists of the cathode lens and the following focusing lens field. The cathode lens, i.e., the homogeneous elec-

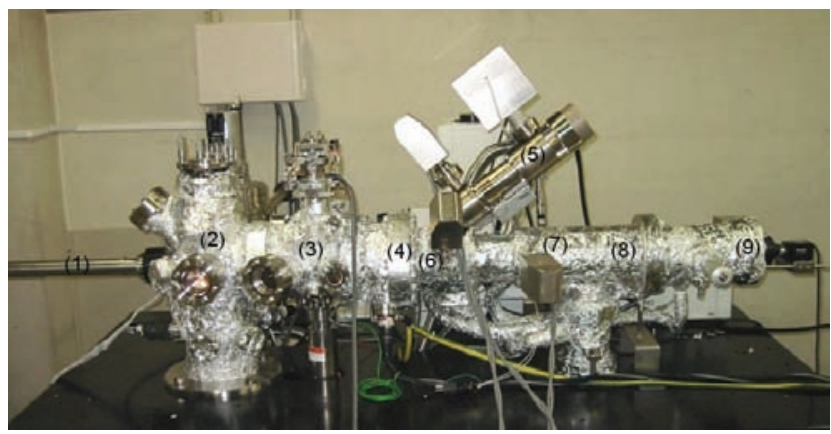

FIG. 3: Electron optical instrument for evaluation of multi emitters. (1) Specimen transfer rod, (2) Specimen preparation chamber, (3) Magnetic-field-superposed cathode lens, (4) Beam Separator (Wien Filter), (5) $\mathrm{LaB}_{6}$ electron gun, (6) Intermediate lens 1, (7) Intermediate lens 2, (8) Projector lens, (9) $\mathrm{MCP} /$ Screen/CCD-camera.

trostatic accelerating field in front of the cathode (= specimen) can form a virtual image of the specimen surface, but not a real image. A focusing lens is, therefore, required after the cathode lens in order to obtain a real image of the specimen surface. According to a theoretical calculation, we have found that for an improvement of the spatial resolution of the cathode lens the electrostatic accelerating field in front of the cathode must be as strong as possible, and superposition of a magnetic field onto a homogeneous electrostatic accelerating field in front of the cathode is greatly effective for decreasing the aberration effect of the following focusing lens field. We have therefore adopted a magnetic-field-superposed cathode lens instead of a pure electrostatic one, as shown in Fig. 2. The electrostatic accelerating field in front of the specimen is typically $5 \mathrm{kV} / \mathrm{mm}$. Similar lens configurations are also proposed by Chmelik et al. [6] and Lenc et al. [7]. Note that in their proposal the magnetic field is only used for the focusing lens field and in not used for superposition onto the homogeneous electrostatic accelerating field in front of the cathode. 
Figure 3 shows an overview picture of the instrument. The whole instrument is evacuated by a combination of ion pumps and turbo molecular pumps, and is capable of high temperature baking to attain an ultra-high vacuum condition of $10^{-8} \mathrm{~Pa}$ for stable operation of multi emitters. The specimens are exchangeable under ultra high vacuum condition using the specimen transfer rod (1). In the specimen preparation chamber (2), heating and aging process of multi emitters, observations of emission patterns from multi emitters, measurements of currentvoltage characteristics and so on are possible before the specimen is brought to the objective lens position (3) by the transfer rod for observation.

\section{IMAGING MODE}

\section{A. SEEM imaging mode}

In the SEEM imaging mode, the electron beam from the $\mathrm{LaB}_{6}$ electron gun, whose accelerating voltage is $10 \mathrm{kV}$, is introduced to the optical axis of the instrument through the beam separator and irradiates the specimen. Then secondary (or reflected) electrons emitted from the specimen are used to image the specimen surface. The beam separator is actually a Wien filter which deflects only the electron beam coming from the $\mathrm{LaB}_{6}$ electron gun. The electrons emitted from the specimen, on the other hand, go straight through the beam separator after being accelerated up to $10 \mathrm{keV}$ and forms the magnified image of the specimen surface onto the entrance plane of the MCP by means of the imaging lens system. The image is intensified by the MCP and is sent to the $\mathrm{PC}$ via $\mathrm{CCD}$ camera.

The incident energy of the electron beam onto the specimen can be controlled to an arbitrary energy by adjusting the output voltage $V_{\mathrm{S}}$ of beam energy adjusting (BEA) unit, where a several tens of $\mathrm{eV}$ is generally used as an incident energy of electron beam for SEEM imaging mode (see Figs. 1 and 2). If the output voltage $V_{\mathrm{S}}$ of BEA unit is kept approximately zero, the incident electron beam cannot reach the specimen and are reflected immediately in front of the specimen, which corresponds to a mirror electron microscope (MEM) imaging mode.

\section{B. PEEM imaging mode}

In the PEEM imaging mode, UV light is irradiated from outside the vacuum chamber through a viewport onto the specimen surface by using a deuterium lamp source. The photo electrons emitted from the specimen surface are accelerated up to $10 \mathrm{keV}$ and are used to form the magnified image of the specimen surface onto the entrance plane of the MCP by the imaging lens system. In this case, the beam separator (= Wien filter) is not used.

\section{FEEM imaging mode}

The FEEM imaging mode is explained below for the case of the Spindt type FEA as a typical example of multi emitters. As the drive voltage (= emitter-to-gate voltage) $V_{\mathrm{d}}$ is gradually increased, some of emitters start to emit field-emitted electrons, which are accelerated up to $10 \mathrm{keV}$ and are imaged by the imaging lens system to form a magnified image of the emission sites of the FEA.

It must be noted that the drive voltage unit (DV unit) should be kept floating at a negative high potential, since the specimen (= multi emitters) is kept floating at a negative high potential of about $-10 \mathrm{kV}$. The floating DV unit is capable of driving multi emitters in DC mode as well as in pulse mode. The frequency and duty ratio (pulse width) can be controlled by a microcomputer from DC to $120 \mathrm{~Hz}$ and 1 to 1/100, respectively, as shown in Fig. 2. The operation mode of the DV unit is very important and will be described later.

\section{Simultaneous observation of SEEM and FEEM images and PEEM and FEEM images}

As mentioned above, the electron optical instrument for evaluation of multi emitters we have developed is a versatile emission microscope which is capable of operating as an SEEM, a PEEM and an FEEM. The most important feature of the instrument is the capability of simultaneous observation of SEEM and FEEM images as well as PEEM and FEEM images in real time and in-situ mode.

The principle of simultaneous observation of SEEM and FEEM images is explained as follows. By irradiating the specimen with electron beam from the $\mathrm{LaB}_{6}$ electron gun, secondary (or reflected) electrons are emitted from the specimen to form a SEEM image. At the same time, if a drive voltage $V_{\mathrm{d}}$ is applied between the emitter and the gate electrode, then a FEEM image is formed by field-emitted electrons from multi emitters and overlaps onto the SEEM image. Thus, simultaneous observation of SEEM and FEEM images is possible.

Simultaneous observation of PEEM and FEEM images can be done in a similar way. By irradiating the specimen with UV light, photo electrons are emitted from the specimen to form a PEEM image. At the same time, if a drive voltage $V_{\mathrm{d}}$ is applied between the emitter and the gate electrode, then a FEEM image is formed by field-emitted electrons from multi emitters and overlaps onto the PEEM image. Thus, simultaneous observation of PEEM and FEEM images is possible.

The SEEM and PEEM images are essentially the magnified images of the specimen surface and hence disclose the surface fine structures of the gate electrode as well as the geometrical shapes of gate holes of FEA. The FEEM image, on the other hand, is essentially the magnified image of the spatial distribution of emission sites of FEA. By simultaneous observation of either SEEM and FEEM images or PEEM and FEEM images, we can therefore distinguish the working emitters from non-working emitters quite obviously. This situation enables us to obtain quantitative knowledge as to the percentage of actually working emitters out of the whole emitters, stability of the emission current from each individual working emitter and so on. 


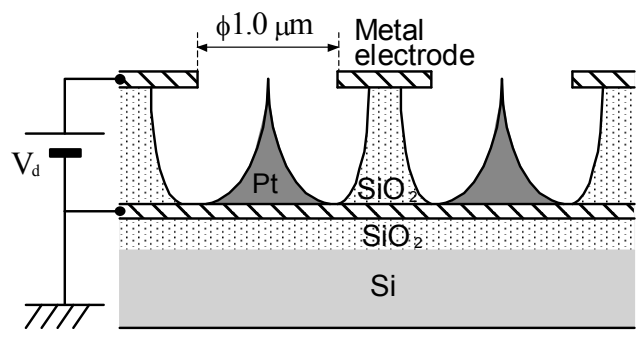

FIG. 4: Cross sectional view of Spindt type FEA without a resistive layer.
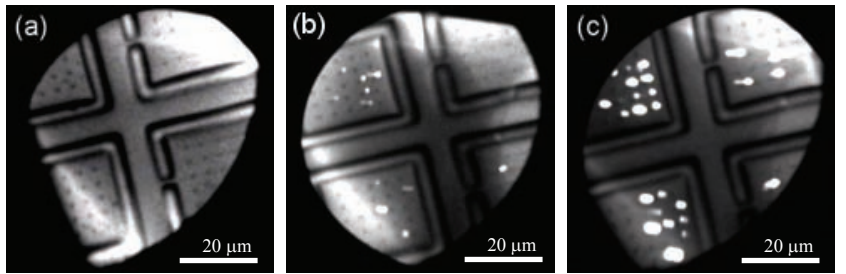

FIG. 5: Simultaneous observation of SEEM and FEEM images in the same area of the FEA under different conditions of drive voltage $V_{\mathrm{d}}$ in $\mathrm{DC}$ mode. (a) At $V_{\mathrm{d}}=0$, no emission. (b) At $V_{\mathrm{d}}=60 \mathrm{~V}$, some of small white spots can be seen, and each small white spot corresponds to each field emission sites and indicates that the corresponding emitter is active. (c) At $V_{\mathrm{d}}=$ $75 \mathrm{~V}$, the number of the emission site as well as the emission current from each emitter is increased.

\section{EXPERIMENTAL RESULTS}

\section{A. Simultaneous observation of SEEM and FEEM images}

The specimen we have observed is a test sample of the Spindt type FEA [8] without a resistance layer as shown in Fig. 4. Therefore, the uniformity of emission characteristics may be rather worse than that of the FEA having a resistance layer. The FEA consists of gate holes of $1 \mu \mathrm{m}$ in diameter and $\mathrm{Pt}$ emitters inside the holes. Gate holes are located at intervals of $5 \mu \mathrm{m}$. The specimen has the size of $3 \times 3 \mathrm{~mm}$ squares and is mounted on a TO5 IC holder for observation. Aging process of the FEA before observation was carried out in the specimen preparation chamber. The FEA was heated at a temperature of $150^{\circ} \mathrm{C}$ for about 3 hours and was driven in DC mode with the drive voltage (= gate voltage) of $60 \mathrm{~V}$ for about 4 hours until the emission current become stable. After aging process, the specimen was brought to the objective lens position by the transfer rod for observation.

Figure 5 shows typical examples of the simultaneous observation of SEEM and FEEM images in the same area of the FEA under different conditions of drive voltage $V_{\mathrm{d}}$ in DC mode. Figure 5(a) shows a typical example of SEEM image at the drive voltage $V_{\mathrm{d}}=0$. Dark spots correspond to gate holes of $1 \mu \mathrm{m}$ in diameter, behind which field emitters are located. When the drive voltage $V_{\mathrm{d}}=$ $60 \mathrm{~V}$ is applied, some of small white spots can be seen as shown in Fig. 5(b), which indicates the FEEM image overlapping onto the SEEM image. Each small white spot
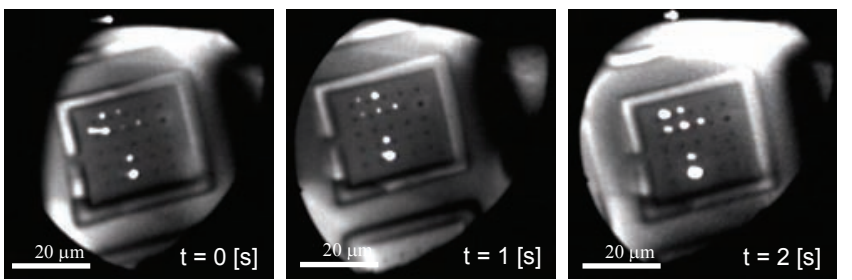

FIG. 6: Simultaneous real time observation of SEEM and FEEM images from the same area of the FEAs under a constant drive voltage of $70 \mathrm{~V}$ in DC mode. The dynamical fluctuations of field emission can be observed as a time series of images.

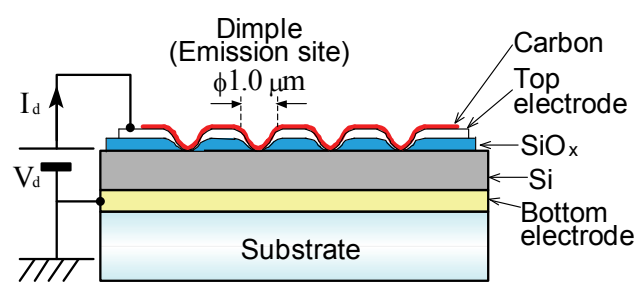

FIG. 7: Cross sectional view of HEED.

corresponds to a field emission site and indicates that the corresponding emitter is active. When the drive voltage $V_{\mathrm{d}}$ is increased up to $75 \mathrm{~V}$, the number of the emission sites as well as the emission current from each emitter is increased, as shown in Fig. 5(c). These results enable us to obtain quantitative knowledge as to the percentage of actually working emitters out of the whole emitters under the respective conditions of drive voltage.

Figure 6 shows typical examples of simultaneous real time observation of SEEM and FEEM images from the same area of the FEA under a constant drive voltage of $70 \mathrm{~V}$ in DC mode. The dynamical fluctuations of field emission can be observed as a series of images for several seconds. This indicates that the instrument is capable of observing instability of the emission current from each individual working emitter and dynamical behaviors of the FEA.

\section{B. Simultaneous observation of PEEM and FEEM images}

The specimen we have used for simultaneous observation of PEEM and FEEM images is a metal-insulatorsemiconductor (MIS) structure device called HEED (highefficiency electron emission device) [1], as shown in Fig. 7. The operating principle of the HEED is summarized as follows. When a drive voltage $V_{\mathrm{d}}$ is applied between the top electrode and the bottom electrode, a diode current Id flows from a top electrode to the bottom electrode, and part of electrons which correspond to several percent of the total diode current are emitted as an emission current from the dimples into vacuum. For detailed description of the HEED, readers should see reference [1]. 

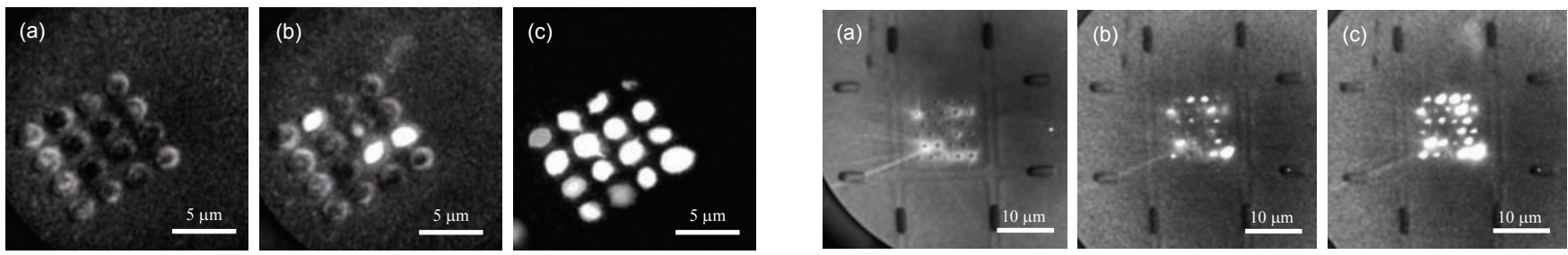

FIG. 8: Simultaneous observation of PEEM and FEEM image in the same area of the HEED under different conditions of drive voltage $V_{\mathrm{d}}$ in $\mathrm{DC}$ mode. (a) At $V_{\mathrm{d}}=0$, no emission. (b) At $V_{\mathrm{d}}=7 \mathrm{~V}$, some of dimples start emission. The electrode surface of the HEED can still be observed as a PEEM image. (c) When the drive voltage $V_{\mathrm{d}}$ is increased up to $11 \mathrm{~V}$, all of $4 \times 4$ dimples are working. However, the PEEM image which indicates the electrode surface of the HEED can no longer be observed.

\section{DC mode operation}

Figure 8 shows typical examples of simultaneous observation of PEEM and FEEM images in the same area of the HEED under different conditions of drive voltage $V_{\mathrm{d}}$ in DC mode.

Figure 8(a) shows a typical example of the PEEM image at the drive voltage $V_{\mathrm{d}}=0$. Sixteen $(=4 \times 4)$ circles in Fig. $8(\mathrm{a})$ correspond to dimples $(1 \mu \mathrm{m}$ in diameter $)$. When the drive voltage $V_{\mathrm{d}}$ is increased to $7 \mathrm{~V}$, some of dimples start emission as shown in Fig. 8(b), where white spots correspond to the FEEM image and overlap onto the PEEM image. The electrode surface of the HEED can still be observed as a PEEM image. When the drive voltage $V_{\mathrm{d}}$ is increased up to $11 \mathrm{~V}$, all of $4 \times 4$ dimples are working as can be seen in Fig. 8(c). However, the PEEM image which indicates the electrode surface of the HEED can no longer be observed because the intensity of the FEEM image formed by electrons emitted from dimples is several orders of magnitude stronger than that of the PEEM image. This situation can be overcome by pulse mode operation of the drive voltage, which will be described in the next section.

\section{Pulse mode operation}

The pulse mode operation of the DV unit allows intermittent operation of multi emitters, and the average value of the emission current is greatly decreased as compared with the case of DC mode operation. This means that even under a sufficiently high drive voltage condition the intensity of the FEEM image might be comparable with that of the PEEM image, and hence the PEEM image, i.e., the surface fine structures of the top electrode would be visible simultaneously with FEEM image.

Figure 9 shows typical examples of simultaneous observation of PEEM and FEEM image in the same area of the HEED under different conditions of the drive voltage $V_{\mathrm{d}}$ in pulse mode. Figure 9(a) shows a typical example of the PEEM image at the drive voltage $V_{\mathrm{d}}=0$. Twenty seven dark spots aligned in a closed packed structure correspond to dimples. The distance between neighboring dimples is $1.9 \mu \mathrm{m}$. When the drive voltage $V_{\mathrm{d}}=10 \mathrm{~V}$ is applied in
FIG. 9: Simultaneous observation of PEEM and FEEM image in the same area of the HEED under different conditions of drive voltage $V_{\mathrm{d}}$ in pulse mode. (a) At $V_{\mathrm{d}}=0$, no emission. (b) At $V_{\mathrm{d}}=10 \mathrm{~V}$ (pulse frequency: $120 \mathrm{~Hz}$, duty ratio: $1 / 100$ ), some small white spots, which correspond to emission sites, appear. (c) At $V_{\mathrm{d}}=12 \mathrm{~V}$ (pulse frequency: $120 \mathrm{~Hz}$, duty ratio: 1/100), all of twenty seven dimples are working, and the PEEM image, i.e., the surface fine structure of the top electrode is also visible.

pulse mode with a pulse frequency of $120 \mathrm{~Hz}$ and a duty ratio of $1 / 100$, some small white spots, which correspond to emission sites, appear, as shown in Fig. 9(b). This indicates that the corresponding dimple is active. When the drive voltage $V_{\mathrm{d}}$ is increased up to $12 \mathrm{~V}$ in pulse mode (pulse frequency: $120 \mathrm{~Hz}$, duty ratio: 1/100), all of 27 dimples are working, and the emission current from each dimple is increased, as shown in Fig. 9(c). In this case the PEEM image, i.e., the surface fine structure of the top electrode is actually visible, while the PEEM image in DC mode operation as shown in Fig. 8(c) cannot be observed.

The pulse mode operation of multi emitters is often required for practical application of multi emitters. Besides the above practical requirement, the pulse mode operation of multi emitters is also very effective for a stable operation of the electron optical instrument for evaluation of multi emitters. As mentioned before, there exists a very strong electrostatic accelerating field between the specimen and the objective lens. If the emission current from the specimen is considerably increased, the vacuum condition in the space between the specimen and the objective lens would become worse. This situation would cause the electric discharge between the specimen and the objective lens. The pulse mode operation of multi emitters greatly reduces the average value of the emission current, and hence is very effective for a stable operation of the instrument without any electric discharge.

\section{DISCUSSIONS}

The instrument enables us to carry out in-situ, real time observation of operating condition of multi emitters and to distinguish the actual working emitters from nonworking emitters. The stability of emission current from each individual emitter can also be monitored. The emission characteristics depend on the emitter surface condition such as contaminations as well as the geometrical condition such as sharpness of the emitter tip. For stable and efficient multi emitters, therefore, we must make clear the reasons why emission current from some of emitters are unstable and why some of emitters are not working. For this purpose the instrument must be equipped with 
surface processing devices such as an ion gun or a plasma source. The resolution of the instrument must also be improved so that sharpness of each individual emitter tip might be estimated. These problems will be the main research subjects in the next stage.

\section{CONCLUSIONS}

We have developed an electron optical instrument for evaluation of multi emitters. The instrument is a versatile emission microscope, in which a magnified image of specimen surface can be obtained by means of electrons emitted from the specimen itself floating at a negative high potential. The instrument is equipped with a beam illumination system that irradiates the specimen with electron beam or UV light for obtaining secondary (or reflected) electrons or photo electrons emitted from specimen surface. Field emitted electrons can also be obtained from emission sites of the specimen by controlling the drive voltage applied through the specimen stage. Thus, the instrument is capable of operating as a secondary electron emission microscope (SEEM), a photo electron emission microscope (PEEM) and a field electron emission micro- scope (FEEM). The most important feature of the instrument is the capability of simultaneous observation of SEEM and FEEM images as well as PEEM and FEEM images in real time and in-situ mode. The operating condition of the multi emitters can be observed in DC mode as well as in pulse mode. Thus, the instrument enables us to obtain quantitative knowledge as to the percentage of actually working emitters out of the whole emitters and to evaluate the stability of the emission current from each individual working emitter.

\section{Acknowledgments}

The authors would like to thank Dr. Akinori Mogami, Dr. Yuji Sakai, Mr. Masato Kudo and Dr. Makoto Kato of JEOL Ltd. for cooperation in developing the electron optical instrument for evaluation of multi emitters. The authors are grateful to Dr. Keiichi Betsui and Dr. Kazunori Inoue of Fujitsu Laboratories Ltd., and Prof. Yasuhito Gotoh of Kyoto University for preparing FEAs. Finally, this work is supported in part by a grant from the Nito Foundation.
[1] N. Negishi, T. Nakada, K. Sakemura, Y. Okuda, H. Satoh, A. Watanabe, T. Yoshikawa, K. Ogasawara, and N. Koshida, J. Vac. Sci. Technol. B 23, 682 (2005).

[2] H. Makishima, S. Miyano, H. Imura, J. Matsuoka, H. Takemura, and A. Okamoto, Appl. Surf. Sci. 146, 230 (1999).

[3] T. H. P. Chang, M. G. R. Thomson, E. Kratschmer, H. S. Kim, M. L. Yu, K. Y. Lee, S. A. Rishton, and B. W. Hussey, J. Vac. Sci. Technol. B 14, 3774 (1996).

[4] T. R. Groves and R. A. Kendall, J. Vac. Sci. Technol. B 16, 3168 (1998).

[5] H. Murata, T. Kimura, Y. Nishimura, H. Shimoyama, A.
Mogami, Y. Sakai, M. Kudo, M. Kato, K. Betsui, and K. Inoue, Technical Digest of the 17th Int. Vacuum Nanoelectronics Conf. (Cambridge, MA), 188 (2004).

[6] J. Chmelik, L. Veneklasen, and G. Marx, Optik 83, 155 (1989).

[7] M. Lenc and I. Mullerova, Ultramicrosc. 45, 159 (1992).

[8] Y. Gotoh, M. Nagao, D. Nozaki, K. Utsumi, K. Inoue, T. Nakatani, T. Sakashita, K. Betsui, H. Tsuji, and J. Ishikawa, J. Appl. Phys. 95, 1537 (2004). 